\title{
Theoretical calculation of photodetachment intensities for $\mathrm{H}_{3} \mathrm{O}^{-}$
}

\author{
Ward H. Thompson, Hans O. Karlsson, and William H. Miller \\ Department of Chemistry, University of California, Berkeley, California 94720 and \\ Chemical Sciences Division, Lawrence Berkeley Laboratory, Berkeley, California 94720
}

(Received 4 June 1996; accepted 28 June 1996)

\begin{abstract}
We have calculated total and arrangement-selected photodetachment intensities for the $\mathrm{H}_{3} \mathrm{O}^{-}$anion (and its deuterated form, $\mathrm{D}_{3} \mathrm{O}^{-}$) using a Green's function in a discrete variable representation with absorbing boundary conditions. A multiply-shifted quasiminimal residual method is used to obtain the Green's function for many energies at once. We present spectra obtained by explicitly treating two and four degrees of freedom. Comparison with experiment indicates that the bending angles in the anion and neutral are more similar than in the current potential energy surfaces. The calculated spectra are also consistent with the suggestion that the barrier should be "earlier." (C) 1996
\end{abstract} American Institute of Physics. [S0021-9606(96)03437-X]

\section{INTRODUCTION}

Photoelectron spectroscopy of negative ions has proven to be a powerful tool for probing the reaction dynamics and the potential energy surface of the corresponding neutral molecular system. ${ }^{1}$ In a typical experiment, the stable anion $A B C^{-}$is photodetached by a fixed freqency laser and the kinetic energy distribution (spectrum) of the detached electron measured. In a fortuitous case, the equilibrium geometry of the anion lies directly below the transition state region of the neutral potential energy surface. Then, in the spirit of transition state theory, one would predict that the spectrum consists of a series of peaks, each corresponding to a state of the activated complex of the $A B C$ neutral and with an intensity proportional to the Franck-Condon overlap of that state with the anion wavefunction. Since the activated complex is not a stable species (decaying to produce either the neutral products or reactants), these states have a finite lifetime which gives rise to broadening of the peaks in the spectrum. Thus the kinetic energy distribution of the detached electron gives detailed information about the transient activated complex which is the gateway to reaction. Comparison with theoretical predictions of the spectrum constitutes a stringent test of an $a b$ initio surface and provides for a detailed assessment of the crucial features of the transition state region which cannot be obtained by a comparison of reactive scattering results.

Calculations for this process were first carried out by Schatz ${ }^{2}$ to study the hydrogen atom exchange reaction between halogen atoms, $\mathrm{X}+\mathrm{HX}^{\prime} \rightarrow \mathrm{XH}+\mathrm{X}^{\prime}$. More recently ${ }^{3}$ comparison of theoretical and experimental results were used to great effect for the $\mathrm{F}+\mathrm{H}_{2}$ reaction, leading to the accurate characterization of the transition state for that reaction. The reaction

$$
\mathrm{OH}+\mathrm{H}_{2} \rightarrow \mathrm{H}_{2} \mathrm{O}+\mathrm{H}
$$

has become the benchmark of choice for quantum treatments of a four-atom reaction, ${ }^{4-14}$ and de Beer et al. ${ }^{15}$ have recently carried out photodetachment experiments and simulations of $\mathrm{H}_{3} \mathrm{O}^{-}$and $\mathrm{D}_{3} \mathrm{O}^{-}$providing information on the potential energy surface of reaction (1.1). Despite the flurry of recent experimental and theoretical work on the $\mathrm{OH}+\mathrm{H}_{2}$ reaction, the best available potential surface ${ }^{16}$ still contains multiple flaws. For example, the surface gives the transition state geometry as a trans configuration though ab initio calculations predict it to be cis; it also contains a spurious well just before the barrier on the $\mathrm{OH}+\mathrm{H}_{2}$ side, which has been partially corrected by Clary. ${ }^{4}$

The photodetachment spectroscopy of $\mathrm{H}_{3} \mathrm{O}^{-}$, however, has some aspects which make it a difficult problem. The anion has two stable geometries, $\mathrm{H}^{-} \cdots \mathrm{H}_{2} \mathrm{O}$ and $\mathrm{HO}^{-} \cdots \mathrm{H}_{2}$ structures, neither of which lies close to the geometry of the neutral transition state. ${ }^{15,17}$ The two anion species lead to distinctly different spectra at different laser polarizations, ${ }^{15}$ and furthermore, excited vibrational states of the anion are delocalized over both geometries and can give FranckCondon overlap with the transition state. An additional difficulty is the lack of knowledge of both the anion and neutral surfaces. The interpretation of the spectra is simplified if one surface is known well (or alternatively, if the spectra are insensitive to one surface), but if both surfaces are not well determined, one is limited to making statements about the similarities and differences between the surfaces.

Section II first reviews the formalism used for calculating total and arrangement-selected photodetachment intensities. Section III discusses the details of the calculations, including the two and four degree of freedom Hamiltonians, the basis set, the neutral and anion potential energy surfaces, the absorbing potentials, and the iterative methods used to obtain the Green's function and the anion bound state. Section IV presents and discusses the results of the photodetachment calculations, and Section V concludes.

\section{REVIEW OF RELEVANT FORMULAS}

The discrete variable representation ${ }^{18-20}$ (DVR) - absorbing boundary conditions (ABC) approach for calculating photodetachment intensities has been presented in detail previously. ${ }^{21}$ Here we wish only to give a brief review of the relevant formulas.

It was previously shown ${ }^{21}$ that the photodetachment in- 
tensity can be obtained in the DVR-ABC formulation within the Franck-Condon approximation by

$$
I(E)=-\frac{1}{\pi} \operatorname{Im} \boldsymbol{\phi}_{b}^{\dagger} \cdot \mathbf{G}^{+}(E) \cdot \boldsymbol{\phi}_{b},
$$

where $\boldsymbol{\phi}_{b}$ is the DVR vector of the bound state of the anion, and the matrix

$$
\mathbf{G}^{+}(E)=(E-\mathbf{H}+i \boldsymbol{\epsilon})^{-1}
$$

is the scattering Green's function with outgoing wave boundary conditions. In the DVR-ABC approach, the " $i \epsilon$," which is added to the energy to enforce the outgoing wave boundary conditions, is allowed to be a function of position. That is, $\epsilon(\mathbf{q})$ is zero in the interaction region and "turns on" in the reactant and product valleys. These absorbing boundary conditions allow the use of an $L^{2}$ basis which is localized in the interaction region to represent the Green's function.

Arrangement-selected photodetachment intensities can also be obtained within the DVR-ABC formulation. ${ }^{21}$ The contribution to the photodetachment intensity due to the neutral dissociating into the arrangement $\gamma=r$ or $p$ is given by

$$
I_{\gamma}(E)=\frac{1}{\pi} \boldsymbol{\phi}_{b}^{\dagger} \cdot \mathbf{G}^{*}(E) \cdot \boldsymbol{\epsilon}_{\gamma} \cdot \mathbf{G}(E) \cdot \boldsymbol{\phi}_{b},
$$

where $\boldsymbol{\epsilon}_{r}\left(\boldsymbol{\epsilon}_{p}\right)$ is the part of the absorbing potential in the reactant(product) arrangement. The total intensity is given by a sum over all arrangements:

$$
I(E)=\sum_{\gamma} I_{\gamma}(E)
$$

Note that both methods require essentially the same amount of computational work since the action of the Green's function onto the vector $\boldsymbol{\phi}_{b}$ is the primary computational task. Thus, the direct and arrangement-selected photodetachment intensities can be computed by Eqs. (2.1) and (2.3) simultaneously with no extra work. Additionally, the intensity obtained from Eq. (2.4) can be compared to that from Eq. (2.1) as a test of the convergence of the Green's function.

\section{DETAILS OF CALCULATION}

\section{A. Jacobi coordinates}

We have chosen to use the Jacobi coordinates of the reactants as shown in Fig. 1; $r_{1}$ and $r_{2}$ denote the $\mathrm{H}_{2}$ and $\mathrm{OH}$ bond distances, respectively, $R$ the distance between the $\mathrm{H}_{2}$ and $\mathrm{OH}$ centers-of-mass, $\gamma_{1}$ the angle between $\mathbf{r}_{1}$ and $\mathbf{R}$, $\gamma_{2}$ the angle between $\mathbf{r}_{2}$ and $\mathbf{R}$, and $\varphi$ the torsional angle.

The calculations treat either two or four degrees of freedom explicitly. In all cases, the $\mathrm{OH}$ bond distance is frozen at its equilibrium value, $r_{2}^{0}=1.8633$ a.u. and the torsional angle is fixed at $\varphi^{0}=0$, i.e., the planar configuration. These values give the proper transition state geometry for the reaction. It was previously observed that calculations of reaction probabilities with these coordinates fixed gave all the qualitative features of a full six degree of freedom treatment. ${ }^{21}$ Here we make no attempt to incorporate the effects of these

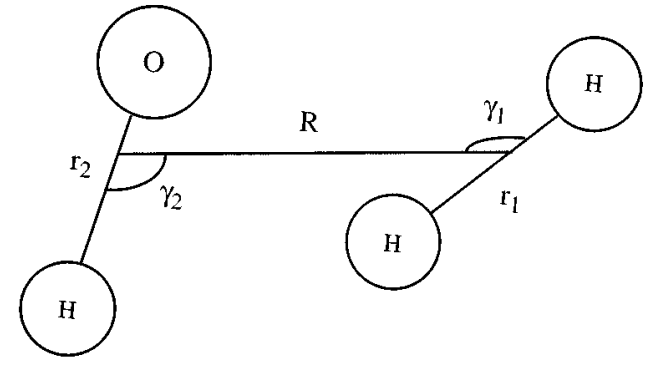

FIG. 1. The reactant Jacobi coordinate system used for the calculations. The dihedral angle, $\varphi$, (not shown) is the out of plane angle between the $\mathrm{OH}$ and $\mathrm{H}_{2}$ bonds.

two coordinates under the assumption that the zero point energies are the same in the anion and neutral.

\section{B. Neutral and Anion Potentials}

Our calculations have used the WDSE potential energy surface for the neutral. This surface is a fit by Schatz and Elgersma ${ }^{16}$ to the $a b$ initio results of Walch and Dunning, ${ }^{22}$ and it also includes a modification by Clary ${ }^{4}$ to remove a spurious well on the $\mathrm{OH}+\mathrm{H}_{2}$ side of the barrier.

For the anion surface we have used the surface of de Beer et al. ${ }^{15}$ which provides a global anharmonic description of the two coordinates $r_{1}$ and $R$. This anion surface has two minima. The global minimum is an $\mathrm{H}^{-} \cdots \mathrm{H}_{2} \mathrm{O}$ structure under the $\mathrm{H}+\mathrm{H}_{2} \mathrm{O}$ (product) valley of the neutral surface. The other local minimum lies under the $\mathrm{OH}+\mathrm{H}_{2}$ (reactant) valley at an $\mathrm{HO}^{-} \cdots \mathrm{H}_{2}$ structure. The energy of the local minimum with the $\mathrm{HO}^{-} \cdots \mathrm{H}_{2}$ geometry is $0.085 \mathrm{eV}$ higher than that of $\mathrm{H}^{-} \cdots \mathrm{H}_{2} \mathrm{O} .{ }^{15}$ The ground state wavefunction on this potential energy surface is localized in the $\mathrm{H}^{-} \cdots \mathrm{H}_{2} \mathrm{O}$ well, while excited states with $\nu \geqslant 2$ are delocalized across both wells. Very little information is available about the potential in the angular (bending) coordinates. Consequently, we use separable harmonic oscillator bending potentials. The frequencies are taken from the $a b$ initio calculations of Xantheas and Dunning. ${ }^{17}$ The equilibrium bending angles are varied, and the effects on the resulting spectra are discussed below.

No fit exists for the anion surface, only the value of the potential on a grid of points in the $r_{1}$ and $R$ coordinates. We have used interpolation to obtain the potential at points within the boundary of the grid. Points outside the boundary are set to a large (constant) value.

\section{Absorbing potential}

The absorbing potential is taken to be a function of the translational Jacobi coordinate in the reactant arrangement, $\epsilon_{r}=\epsilon_{r}(R)$, and the $\mathrm{H}_{2}$ bond distance in the product arrangement $\epsilon_{p}=\epsilon_{p}\left(r_{1}\right)$. There are several satisfactory choices for the functional form of the absorbing potential. However any choice must turn on slowly enough not to cause reflection, yet be strong enough to absorb all outgoing flux. We have found the quartic potential to work well, 


$$
\epsilon_{\gamma}\left(R_{\gamma}\right)=\lambda\left(\frac{R_{\gamma}-R_{0, \gamma}}{R_{\max , \gamma}-R_{0, \gamma}}\right)^{4},
$$

where $\gamma=p, r$ is the arrangement index and $R_{p}=r_{1}$, $R_{r}=R . R_{0, \gamma}$ and $R_{\max , \gamma}$ are the starting and ending points of the absorbing potential in the $\gamma$ arrangement. $\lambda$ is a strength parameter representing the maximum value of the absorbing potential, generally it is taken to be about $2 \mathrm{eV}$. The beginning of the absorbing strip is chosen such that the imaginary potential has significant value only where the interaction potential is small.

\section{Two degrees of freedom}

The simplest meaningful calculation treats $r_{1}$ and $R$ explicitly while the other four coordinates are fixed. In this case, the Hamiltonian is given by

$$
\hat{H}=-\frac{\hbar^{2}}{2 \mu_{1}} \frac{\partial^{2}}{\partial r_{1}^{2}}-\frac{\hbar^{2}}{2 \mu_{R}} \frac{\partial^{2}}{\partial R^{2}}+\hat{V}\left(r_{1}, R ; r_{2}^{0}, \gamma_{1}^{0}, \gamma_{2}^{0}, \varphi^{0}\right)
$$

where $r_{2}^{0}, \gamma_{1}^{0}, \gamma_{2}^{0}$, and $\varphi^{0}$ represent the fixed values of these coordinates. This Hamiltonian represents both the $\mathrm{OH}+\mathrm{H}_{2}$ and $\mathrm{H}_{2} \mathrm{O}+\mathrm{H}$ arrangements. From these calculations the effect of the $\mathrm{H}_{2}$ and $\mathrm{HO}-\mathrm{H}$ stretches on the photodetachment spectrum is obtained.

We have used the sinc-function DVR of Colbert and Miller $^{23}$ for the $r_{1}$ and $R$ coordinates. The DVR basis ${ }^{20}$ has the advantages that the potential energy is approximated as a diagonal matrix and the Hamiltonian matrix is sparse for a multidimensional problem. The former means that no integrals need to be evaluated numerically to obtain the matrix elements of the potential - the diagonal elements are simply the potential evaluated at the DVR grid point. The DVR matrix elements of the absorbing potential are also diagonal and similarly evaluated. The sparsity allows the linear algebra to be solved using iterative methods (see Section III F) which makes large dimensional problems tractable when one cannot store the entire Hamiltonian matrix. The radial kinetic energy matrix elements can be expressed in closed form ${ }^{23}$ as

$$
\begin{aligned}
\left(\hat{T}_{R}\right)_{i, i^{\prime}}= & \frac{\hbar^{2}}{2 \mu_{R} \Delta R^{2}}(-1)^{\left(i-i^{\prime}\right)} \\
& \times\left\{\begin{array}{ll}
\pi^{2} / 3-1 / 2 i^{2}, & i=i^{\prime} \\
\frac{2}{\left(i-i^{\prime}\right)^{2}}-\frac{2}{\left(i+i^{\prime}\right)^{2}}, & i \neq i^{\prime}
\end{array}\right\},
\end{aligned}
$$

for the $R$ coordinate, and similarly for $r_{1}$. In practice, a direct product (raw) grid is first laid down in these coordinates. The "refined" grid is then obtained by truncating the raw grid according to two criterion: (1) an energy cuttoff, i.e., if the potential at a given DVR point is greater than some value $V_{\text {cut }}$, then that point is discarded, and (2) the boundaries of the absorbing potential in the reactant and product valleys. Then the matrix elements of the Hamiltonian are computed in the DVR. Finally, the linear system

$$
(E \mathbf{I}-\mathbf{H}+i \boldsymbol{\epsilon}) \cdot \mathbf{x}=\boldsymbol{\phi}_{b},
$$

is solved for

$$
\mathbf{x}=\mathbf{G}^{+}(E) \cdot \boldsymbol{\phi}_{b},
$$

which is used to evaluate Eqs. (2.1) and (2.3).

\section{E. Four degrees of freedom}

The four degree of freedom Hamiltonian explicitly treats the $r_{1}, R, \gamma_{1}$, and $\gamma_{2}$ coordinates and is given by

$$
\begin{aligned}
\hat{H}= & -\frac{\hbar^{2}}{2 \mu_{1}} \frac{\partial^{2}}{\partial r_{1}^{2}}-\frac{\hbar^{2}}{2 \mu_{R}} \frac{\partial^{2}}{\partial R^{2}}+\left(\frac{1}{2 \mu_{1} r_{1}^{2}}+\frac{1}{2 \mu_{R} R^{2}}\right) \hat{\mathbf{j}}_{1}^{2} \\
& +\left(\frac{1}{2 \mu_{2} r_{2}^{2}}+\frac{1}{2 \mu_{R} R^{2}}\right) \hat{\mathbf{j}}_{2}^{2}+\hat{V}\left(r_{1}, R, \gamma_{1}, \gamma_{2} ; r_{2}^{0}, \varphi^{0}\right),
\end{aligned}
$$

where $\hat{\mathbf{j}}_{1}^{2}$ and $\hat{\mathbf{j}}_{2}^{2}$ are the angular momentum operators for the $\mathrm{H}_{2}$ and $\mathrm{OH}$ bonds, respectively. The radial sinc-function DVR is used as described in Section III D. A GaussLegendre DVR is used for the $\gamma_{2}$ angle. The $1 D$ kinetic energy matrix elements for the angular DVR of the $\hat{\mathbf{j}}_{2}^{2}$ operator are then given by a sum over Legendre polynomials:

$$
\begin{aligned}
\left(\hat{\mathbf{j}}_{2}^{2}\right)_{i, i^{\prime}}= & \sum_{j=0}^{N-1} \sqrt{w_{i}} P_{j}\left(\cos \gamma_{2 i}\right) j(j+1) \hbar^{2} \\
& \times \sqrt{w_{i^{\prime}}} P_{j}\left(\cos \gamma_{2 i^{\prime}}\right),
\end{aligned}
$$

where $N$ is the number of angular DVR points. A symmetrized Gauss-Legendre DVR is used for the $\gamma_{1}$ angle. Exploiting the exchange symmetry of $\mathrm{H}_{2}$ means only half the angular DVR points are needed for $\gamma_{1}$ for a given parity. The symmetrized matrix elements are given by

$$
\begin{aligned}
\left(\hat{\mathbf{j}}_{1}^{2}\right)_{i, i^{\prime}}^{p}= & \sum_{j=0}^{N-1} \sqrt{w_{i}} P_{j}\left(\cos \gamma_{1 i}\right) j(j+1) \hbar^{2} \\
& \times \frac{1}{\sqrt{2}}\left[1+(-1)^{(p+j)}\right] \sqrt{w_{i^{\prime}}} P_{j}\left(\cos \gamma_{1 i^{\prime}}\right),
\end{aligned}
$$

where $p$ is the parity quantum number which is either even or odd.

As mentioned above, the $r_{2}$ and $\varphi$ coordinates are not expected to have a significant impact on the photodetachment spectrum. Thus the spectrum calculated with this Hamiltonian should represent the best theoretical prediction of the experimental spectrum (at least for the purposes of this paper). The comparison of the calculated spectrum with the experimental result then gives information about the accuracy (or inaccuracy) of the neutral and anion potential energy surfaces.

\section{F. Iterative Methods}

\section{Acting the Green's function}

To calculate the photodetachment spectra one needs to evaluate the action of a Green's operator onto $\boldsymbol{\phi}_{\mathbf{b}}$, the anion bound state,

$$
\mathbf{G}^{+}(E) \cdot \boldsymbol{\phi}_{\mathbf{b}}=\mathbf{x}
$$

which can be done by solving the linear system 


$$
(E-\mathbf{H}+i \boldsymbol{\epsilon}) \cdot \mathbf{x}=\boldsymbol{\phi}_{\mathbf{b}} .
$$

For multi-dimensional problems the size of the Hamiltonian is too large to be stored in core memory, the only practical operation being matrix-vector multiplication. We have had good experience using Krylov subspace methods, especially the generalized minimal residual ${ }^{24}$ (GMRES) and the quasiminimal residual ${ }^{25}$ (QMR) methods. The GMRES method, which is based on the Arnoldi algorithm, explicitly orthogonalizes the generated Krylov vectors, leading to a storage need and computational time growing linearly with the number of iterations. In practice GMRES has to be restarted every $m$ th iteration, which may slow down the convergence rate. The QMR method is based on the Lanczos algorithm, with a constant computational cost and storage need per iteration. Only about six vectors need to be stored. The drawback is that orthogonality is lost between the Lanczos vectors and the convergence will slow down. Both GMRES and QMR have shown to be of great use for photodetachment and reactive scattering problems with complex symmetric Hamiltonians. ${ }^{14,26}$

A drawback is that the linear system (3.10) has to be solved for several energies, or shifts, $E$. An optimal approach would be if we were able to use methods similar to GMRES and QMR, and modify them to handle several shifts at once. This is indeed possible. Here we have used QMR (Refs. 25, 27) due to its more favorable scaling for storage and computational cost, allowing for the study of a very large system, but the main idea is valid also for GMRES. ${ }^{24,28}$

An important property of the Krylov space

$$
K_{m}(\mathbf{A}, \mathbf{b})=\left\{\mathbf{b}, \mathbf{A b}, \mathbf{A}^{\mathbf{2}} \mathbf{b}, \ldots, \mathbf{A}^{\mathbf{m}-\mathbf{1}} \mathbf{b}\right\}
$$

is that it is invariant under shifts, i.e.

$$
K_{m}(\mathbf{A}, \mathbf{b})=K_{m}(\mathbf{A}-E \mathbf{I}, \mathbf{b}),
$$

where $\mathbf{I}$ is a unit matrix and $E$ a scalar shift. By using this property in conjunction with QMR a multiply-shifted quasiminimal residual ${ }^{27}$ (MSQMR) can be derived. Each iteration in MSQMR consists of two types of calculations: computations that build common Krylov space information and "private" computations for the $s$ different shifts. Computations of the first kind, essentially the Lanczos algorithm, increases the dimension of the Krylov space with one per iteration and consists of a matrix-vector multiplication, two dot products and three vector updates. For each shift we need to update four vectors and a small number of scalar quantities. Note that the computationally most demanding part, the matrix-vector multiplication, is done only once for all shifts. A sketch of the MSQMR for solving a system with $s$ shifts is as follows: ${ }^{27}$

(0) For $j=1,2, \ldots s$ set $\mathbf{x}_{\mathbf{0}}^{(\mathbf{j})}=0$ and $\mathbf{r}_{\mathbf{0}}^{(\mathbf{j})}=b$.

$\operatorname{Set} \mathbf{v}_{\mathbf{1}}=\mathbf{b} /\|\mathbf{b}\|$.

For $n=1,2, \ldots$, do:

(1) Perform the $n$th iteration of the Lanczos algorithm. This gives matrices $\mathbf{V}_{\mathbf{n}}, \mathbf{V}_{\mathbf{n}+\mathbf{1}}$ and $\mathbf{T}_{\mathbf{n}}$ with $\mathbf{A} \mathbf{V}_{\mathbf{n}}=\mathbf{V}_{\mathbf{n}+\mathbf{1}} \mathbf{T}_{\mathbf{n}}$, where $\mathbf{V}_{\mathbf{n}}$ is a matrix of Lanczos vectors and $\mathbf{T}_{\mathbf{n}}$ is the tri-diagonal Lanczos matrix;
For all $j=1,2, \ldots, s$ for which $x_{n}^{(j)}$ has not converged yet:

(2) Update the QR factorization

$$
\mathbf{T}_{\mathbf{n}}^{(\mathbf{j})}=\left(\mathbf{Q}_{\mathbf{n}}^{(\mathbf{j})}\right)^{\mathbf{H}}\left[\begin{array}{c}
\mathbf{R}_{\mathbf{n}}^{(\mathbf{j})} \\
0
\end{array}\right]
$$

of the upper Hessenberg matrix

$$
\mathbf{T}_{\mathbf{n}}^{(\mathbf{j})}=\mathbf{T}_{\mathbf{n}}-E_{j}\left[\begin{array}{c}
\mathbf{I}_{\mathbf{n}} \\
0
\end{array}\right] .
$$

(3) Update the vector

$$
\left[\begin{array}{c}
\tau_{1}^{(j)} \\
\vdots \\
\tau_{n}^{(j)}
\end{array}\right]=\mathbf{Q}_{\mathbf{n}}^{(\mathbf{j})}\left[\begin{array}{c}
\|b\| \\
0 \\
\vdots \\
0
\end{array}\right] .
$$

(4) Update the vector

$$
p_{n}^{(j)}=\mathbf{V}_{\mathbf{n}}\left(\mathbf{R}_{\mathbf{n}}^{(\mathbf{j})}\right)^{-1}\left[\begin{array}{c}
0 \\
\vdots \\
0 \\
1
\end{array}\right] \text {. }
$$

(5) Compute

$$
x_{n}^{(j)}=x_{n-1}^{(j)}+\tau_{n}^{(j)} p_{n}^{(j)} .
$$

(6) If all $x_{n}^{(j)}$ have converged: STOP.

Convergence is checked using an upper residual bound, which is a good upper bound to the true error. ${ }^{25}$ Usually iterative Krylov subspace methods are combined with preconditioners to improve the convergence rate. Unfortunately, standard preconditioners destroy the special structure of the shifted system, and can thus not be used (with the probable exception of polynomial preconditioning). This is normally of little concern since the speedup gained by solving $s$ problems with an un-preconditioned MSQMR is by far larger than solving $s$ individual preconditioned linear systems.

The two degree of freedom photodetachment intensities shown were calculated for 300 energies at one time. The CPU time on a RISC/6000 Model 590 was approximately 2 $\min$. This represents a reduction by a factor of at least 40 in CPU time as compared to running each energy independently. The four degree of freedom intensities were calculated at about 130 energies. The total energy range was broken up into four or five segments for which up to 50 energies were computed at one time. These smaller energy ranges were required by the larger spectral range of the 4D Hamiltonian which slows convergence.

\section{Obtaining the anion bound state}

We have used a Lanczos scheme applicable to a fully coupled potential to obtain the bound state wavefunction. A new Hamiltonian is formed, $\hat{H}_{\text {anion }}$ which has the same kinetic energy as $\hat{H}$ but the neutral potential is replaced by $V_{\text {anion }}\left(r_{1}, R, \gamma_{1}, \gamma_{2} ; \gamma_{1}^{0}, \gamma_{2}^{0}\right)$. Here $\gamma_{1}^{0}$ and $\gamma_{2}^{0}$ are the equilibrium bending angles for the anion. Note that this Hamil- 
tonian is real symmetric. A Krylov space is formed with reorthogonalization to all previous vectors. The Lanczos algorithm is used to obtain the lowest eigenvalues and eigenvectors. (Typically the number of Lanczos iterations needed is between 100 and 140.) The eigenvectors are examined to determine the vibrational state in the coupled $r_{1}$ and $R$ coordinates.

\section{RESULTS AND DISCUSSION}

Recently, de Beer et al. ${ }^{15}$ presented experimental photodetachment spectra of $\mathrm{H}_{3} \mathrm{O}^{-}$and $\mathrm{D}_{3} \mathrm{O}^{-}$as well as two degree of freedom simulation results. The experimental spectra were taken at two laser polarizations $\theta=0^{\circ}$ and $\theta=90^{\circ}$. The $\theta=0^{\circ}$ spectra was attributed to the $\mathrm{H}^{-} \cdots \mathrm{H}_{2} \mathrm{O}$ anion structure and consist of three peaks at electron binding energies of $1.53,2.00$, and $2.38 \mathrm{eV}$. (For $\mathrm{D}^{-} \cdots \mathrm{D}_{2} \mathrm{O}$ the peaks occur at $1.53,1.88$, and $2.17 \mathrm{eV}$.) These peaks were assigned to the stretching progression of the $\mathrm{OH}(\mathrm{OD})$ local mode stretch in the neutral and denoted (000),(001), and (002), respectively. [This is the notation for $\mathrm{H}_{2} \mathrm{O}$ vibrations: $\left(\nu_{1}, \nu_{2}, \nu_{3}\right)$ where $\nu_{1}$ is the symmetric stretch, $\nu_{2}$ the bend, and $\nu_{3}$ the antisymmetric stretch quantum number.] The peak lineshapes have some asymmetry indicating possible excitation of the $\nu_{2}$ bending mode. The $\theta=90^{\circ}$ spectra are qualitatively different with broad peaks at 1.53 and $2.05 \mathrm{eV}$. These peaks are not shifted upon deuteration.

The simulations of de Beer et al. ${ }^{15}$ explicitly treated the $\mathrm{HO}-\mathrm{H}$ and $\mathrm{H}-\mathrm{H}$ bonds. They performed ab initio calculations to obtain the anion potential as a function of these two coordinates. Their simulated spectra reproduce the main features of the experimental results. The $\theta=0^{\circ}$ spectra correspond to the photodetachment from the $v=0$ state of the anion which is completely localized in the $\mathrm{H}^{-} \cdots \mathrm{H}_{2} \mathrm{O}$ well. In contrast, the $\theta=90^{\circ}$ spectra are attributed to the $v=2$ state of the anion; this is the first state delocalized across both geometries of the anion. The peak at $2.05 \mathrm{eV}$ is due to excitation from the $\mathrm{HO}^{-} \cdots \mathrm{H}_{2}$ structure. In this section we present the theoretical photodetachment spectra obtained from explicitly treating two and four degrees of freedom as described in Sections III D and III E.

\section{A. Two degrees of freedom}

We have performed the two degree of freedom calculations described in Section III D for the few lowest eigenstates of the anion. The purpose of presenting two degree of freedom results here is twofold: (1) to show that, though the coordinates and method are different, our results are consistent with those of de Beer et al., ${ }^{15}$ and (2) to illustrate the basic features of the photodetachment spectra which can be compared with higher dimensional results.

Figure 2 shows the 2D total and arrangement-selected photodetachment spectra from the $\nu=0$ state of the anion for (a) $\mathrm{H}_{3} \mathrm{O}^{-}$, and (b) $\mathrm{D}_{3} \mathrm{O}^{-}$. Note that the energy of the separated reactants $\mathrm{OH}+\mathrm{H}_{2}$ is the zero of energy on the neutral potential energy surface. The lowest possible scattering energy is then $-0.64 \mathrm{eV}$ which is the exoergicity of the reaction. ${ }^{16}$ For these calculations the $\mathrm{OH}$ and $\mathrm{H}_{2}$ angles were
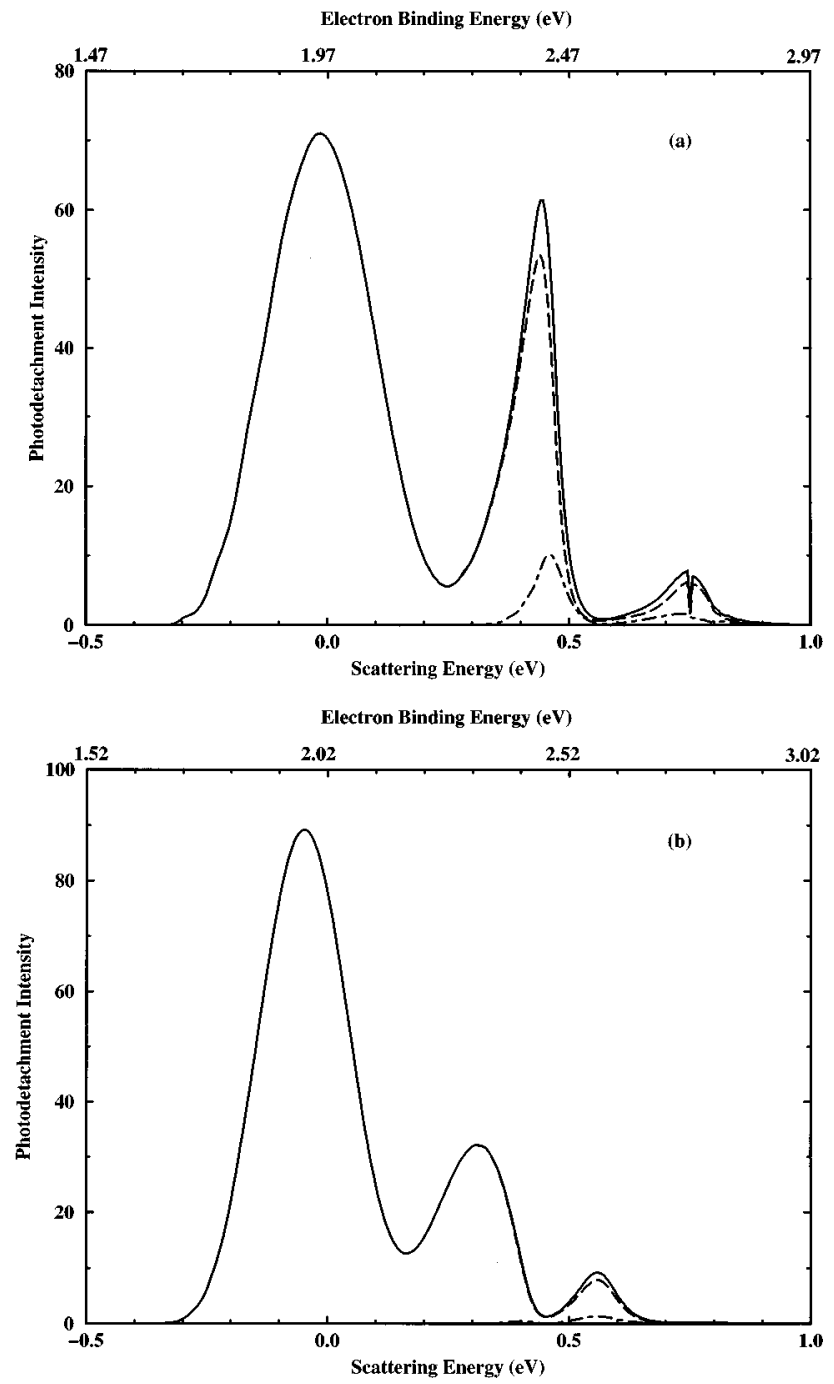

FIG. 2. Two degree of freedom photodetachment intensities from the $\nu=0$ state of the anion for (a) $\mathrm{H}_{3} \mathrm{O}^{-}$and (b) $\mathrm{D}_{3} \mathrm{O}^{-}$. The solid line is the total intensity, the dashed line is the product-selected intensity, and the dot-dashed line is the reactant-selected intensity.

set equal to the equilibrium values for the $\mathrm{H}^{-} \cdots \mathrm{H}_{2} \mathrm{O}$ structure, $\gamma_{1}^{0}=175.9^{\circ}$ and $\gamma_{2}^{0}=104.8^{\circ}$.

Recall that the $\nu=0$ anion state is localized under the $\mathrm{H}+\mathrm{H}_{2} \mathrm{O}$ valley. These spectra show the three peaks attributed to the local mode $\mathrm{OH}(\mathrm{OD})$ stretch in the neutral $\mathrm{H}_{2} \mathrm{O}$ $\left(\mathrm{D}_{2} \mathrm{O}\right)$. The experimentally observed $(000) \rightarrow(001)$ and $(001) \rightarrow(002)$ peak spacings are 0.47 and $0.38 \mathrm{eV}(0.35$ and $0.29 \mathrm{eV}$ ) for the $\mathrm{H}_{3} \mathrm{O}^{-}$(deuterated) spectrum. Our calculated spectra are in good agreement giving spacings of 0.46 and $0.30 \mathrm{eV}$ (0.36 and $0.25 \mathrm{eV}$ deuterated).

These spectra are in reasonable agreement with the simulations of de Beer et al. ${ }^{15}$ indicating our choice of somewhat different coordinates is not significant. The primary difference is the relative intensity of the (001) peak in the $\mathrm{H}_{3} \mathrm{O}^{-}$spectrum is larger than they observed. (Both simulations show a larger relative intensity for this peak than seen in the experiment.) Another difference is the dip in the 
(002) peak of the $\mathrm{H}_{3} \mathrm{O}^{-}$spectrum. This dip appears at the energy of the $\mathrm{H}_{2}(v=1)$ vibrational level.

The arrangement-selected intensities show that most of the intensity leads to the formation of $\mathrm{H}+\mathrm{H}_{2} \mathrm{O}$ products. This would be expected from the position of the Franck-Condon region. However, we do see some intensity leading to the formation of the $\mathrm{OH}+\mathrm{H}_{2}$ reactants. Since there is no Franck-Condon overlap on the reactant side of the barrier in this case this must occur by passing over the barrier. For the deuterated case virtually no reactants are formed. This can be explained by the fact that the energy local OD stretch levels are lower in energy thus most of the intensity is energetically forbidden from passing over the barrier.

The results of the experiment are given in electron binding energy $(e B E)$. The conversion to the $e B E$ from the scattering energy, $E$, is given by

$$
e B E=E+E_{A}+D_{e}-E_{\nu}^{-},
$$

where $E_{A}$ is the electron affinity of $\mathrm{OH}, D_{e}$ is the dissociation energy of $\mathrm{H}^{-}\left(\mathrm{H}_{2} \mathrm{O}\right)$ to obtain $\mathrm{HO}^{-}+\mathrm{H}_{2}$, and $E_{\nu}^{-}$is the vibrational energy of the anion. de Beer et al. ${ }^{15}$ give $E_{A}=1.83 \mathrm{eV}$ and $D_{e}=0.325 \mathrm{eV}$. Alternatively, the $e B E$ can also be calculated by using the electron affinity of $\mathrm{H}, 0.75$ $\mathrm{eV}$, and the dissociation energy of $\mathrm{H}^{-}\left(\mathrm{H}_{2} \mathrm{O}\right)$ to give $\mathrm{H}_{2} \mathrm{O}+\mathrm{H}^{-}$, calculated to be $0.79 \mathrm{eV}$. This gives a slightly different number by about $0.03 \mathrm{eV}$. (We have used the first method.) The uncertainty lies in the dissociation energies as discussed by de Beer et al.. ${ }^{15}$ We will address this in Section IV B. As seen from Fig. 2, our calculations give the first peak centered at $1.95 \mathrm{eV}$ and $1.97 \mathrm{eV}$ for $\mathrm{H}_{3} \mathrm{O}^{-}$and $\mathrm{D}_{3} \mathrm{O}^{-}$, respectively. This compares to the experimental value of $1.53 \mathrm{eV}$ for both. We will discuss this discrepancy in Section IV B.

The photodetachment spectra for (a) $\mathrm{H}_{3} \mathrm{O}^{-}$and (b) $\mathrm{D}_{3} \mathrm{O}^{-}$are shown in Figure 3 for the $\nu=1$ state of the anion. The bending angles are the same as in Fig. 2. The spectra are similar to those with $\nu=0$ except the (000) peak is split into two. The (001) peak now leads to a greater fraction of reactants formed in the case of $\mathrm{H}_{3} \mathrm{O}^{-}$, and some formation of reactants for $\mathrm{D}_{3} \mathrm{O}^{-}$. In addition, we see sharp features in the reactant spectrum at the energy that the reactants become energetically accessible and at the energy of $\mathrm{H}_{2}(v=1)$.

Figure 4 is the same as Figs. 2 and 3 for the $\nu=2$ state of the anion. These spectra are significantly different from those for $\nu=0$ and $\nu=1$. This anion wavefunction is delocalized over both the $\mathrm{H}^{-} \cdots \mathrm{H}_{2} \mathrm{O}$ and $\mathrm{HO}^{-} \cdots \mathrm{H}_{2}$ wells. The product-selected spectra resemble somewhat that for the $\nu=1$ state in Fig. 3. The spectra's dominating features are sharp and lead to the formation of reactants. This is in qualitative agreement with the results of de Beer et al. ${ }^{15}$ The primary difference is the relative heights of the sharp peak at $0.29 \mathrm{eV}$ and the broader peak centered around $0.4 \mathrm{eV}$. This sharp peak was attributed by de Beer et al. to the spurious well on the reactant side of the barrier. The feature at 0.75 $\mathrm{eV}$ (the energy of $\mathrm{H}_{2}(v=1)$ ) is observed in both calculations. We note that the broad peak is centered around 2.20
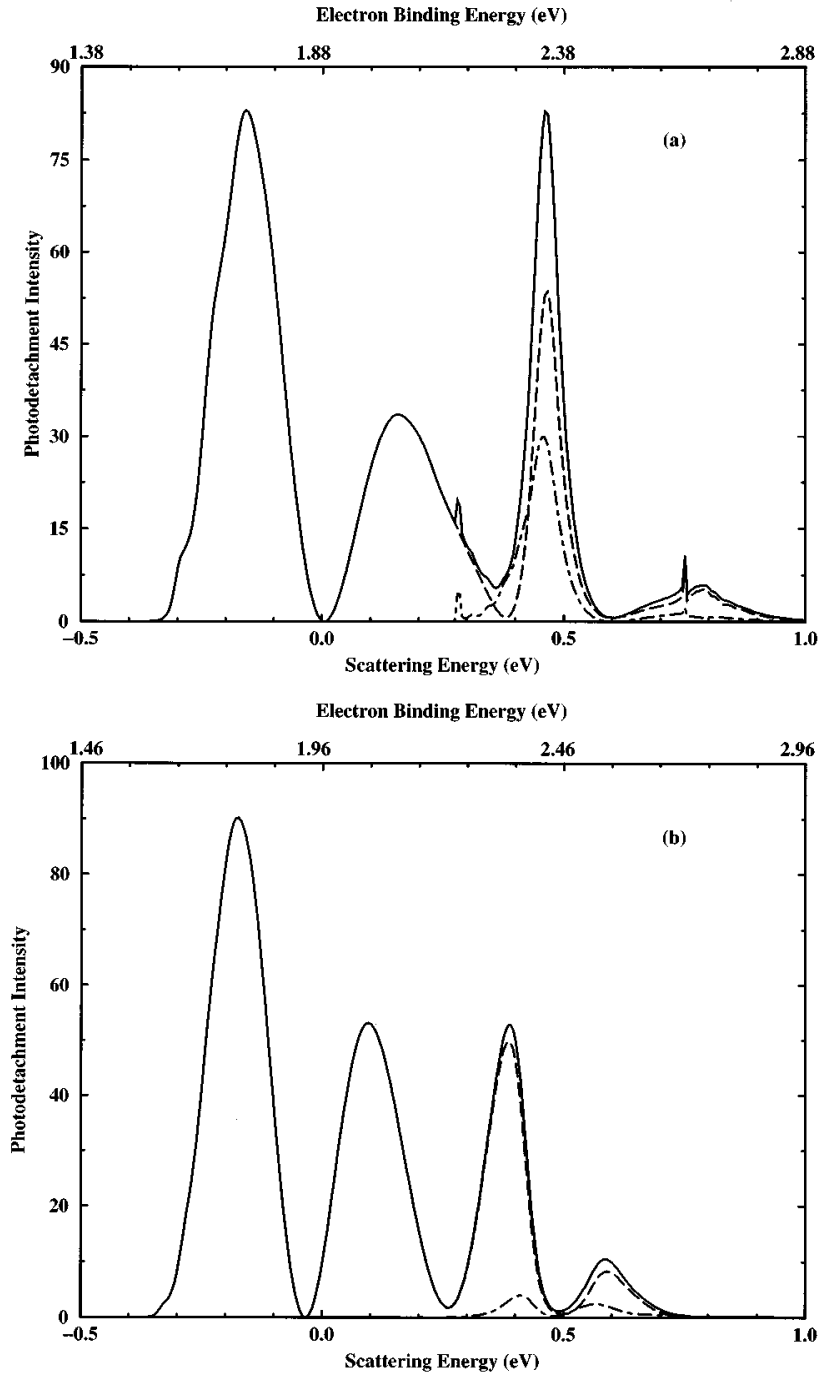

FIG. 3. Same as Fig. 2 but for the $\nu=1$ state of the anion.

and $2.30 \mathrm{eV}$ electron binding energy for $\mathrm{H}_{3} \mathrm{O}^{-}$and $\mathrm{D}_{3} \mathrm{O}^{-}$, respectively, compared with $2.05 \mathrm{eV}$ as observed in the experiment.

\section{B. Four degrees of freedom}

Here we present calculations of the photodetachment intensity explicitly treating four degrees of freedom including the $\mathrm{H}_{2}$ and $\mathrm{OH}$ bending angles $\gamma_{1}$ and $\gamma_{2}$ as described in Section III E. The equilibrium bending angles used for the anion potential are varied and we discuss the effect on the spectrum. All calculations were done for even parity $(p=0)$.

Figure 5 shows the $\nu=0$ photodetachment spectra for (a) $\mathrm{H}_{3} \mathrm{O}^{-}$and (b) $\mathrm{D}_{3} \mathrm{O}^{-}$with anion equilibrium angles $\gamma_{1}^{0}=175.9^{\circ}$ and $\gamma_{2}^{0}=104.8^{\circ}$. The spectra are significantly different from the corresponding $2 \mathrm{D}$ results. The $\mathrm{H}_{3} \mathrm{O}^{-}$spectrum consists of the (000) and (001) peaks with a spacing of $0.45 \mathrm{eV}$ but the peaks are much broader than in the 2D spectrum (Fig. 2) and there is superimposed structure due to excitation of the bending degrees of freedom. The (002) peak may be hidden by the bending progression off the 

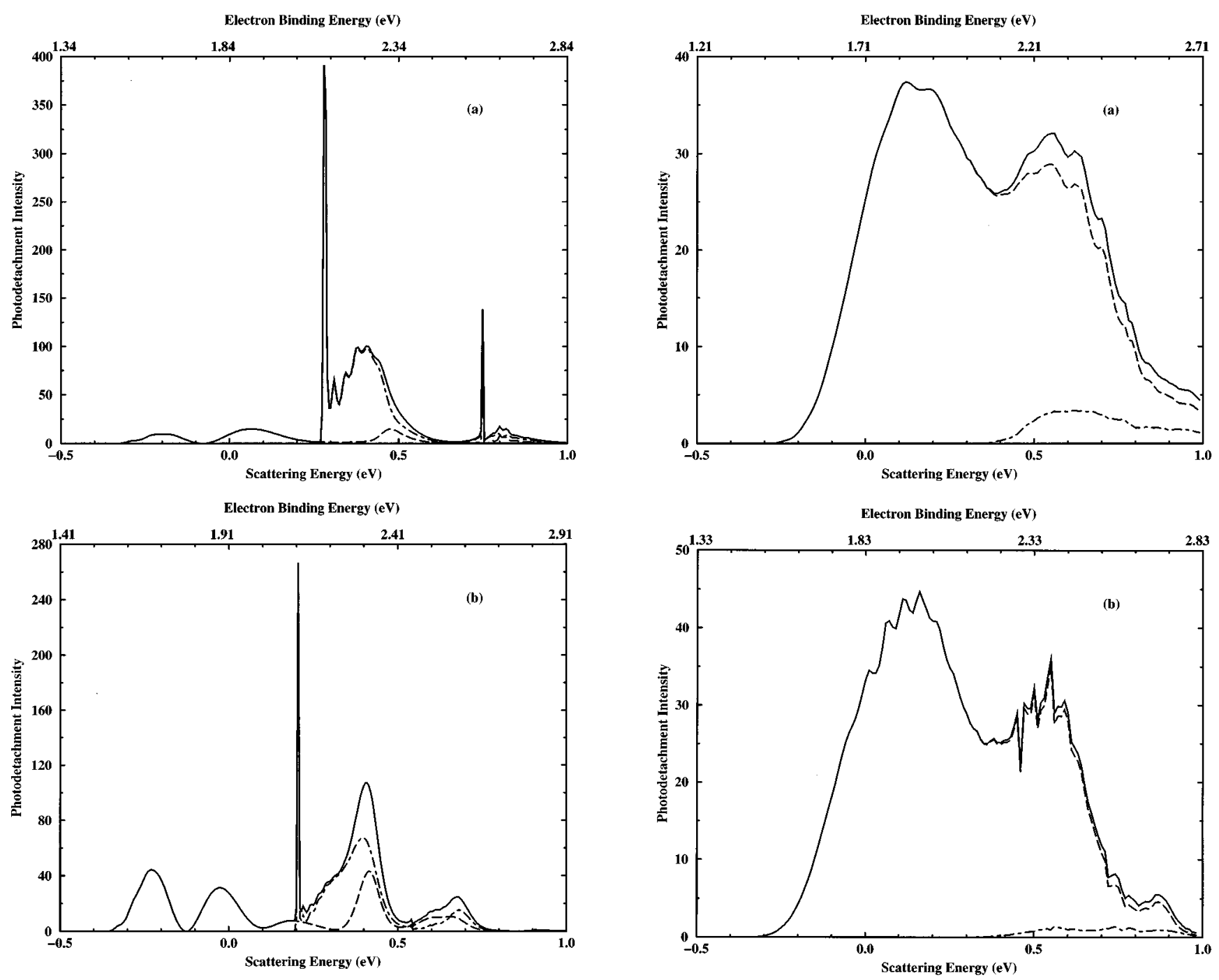

FIG. 4. Same as Fig. 2 but for the $\nu=2$ state of the anion.

(001) peak. The relative peak intensities are virtually the same as in the 2D case; the (000) peak is only slightly higher than the (001) peak. The reactant-selected intensity is small as in the $2 \mathrm{D}$ spectrum but is now a single broad feature. In the deuterated spectrum all three peaks are observed with $(000) \rightarrow(001)$ peak spacings of 0.39 and $0.32 \mathrm{eV}$, respectively. These peak spacings are larger than those found in the $2 \mathrm{D}$ calculations. As in the case of $\mathrm{H}_{3} \mathrm{O}^{-}$the peaks are broadened with superimposed structure not seen in the $2 \mathrm{D}$ spectrum. The relative intensity of the (001) peak to the (000) peak is larger than that seen in the $2 \mathrm{D}$ results. Only a small fraction of the intensity leads to the formation of the reactants.

In Fig. 6 we show the $\nu=0$ spectrum with equilibrium angles $\gamma_{1}^{0}=169.0^{\circ}$ and $\gamma_{2}^{0}=122.0^{\circ}$ for (a) $\mathrm{H}_{3} \mathrm{O}^{-}$and (b) $\mathrm{D}_{3} \mathrm{O}^{-}$. These angles correspond to the transition state geometry of the neutral. The three peaks are observed as in the $2 \mathrm{D}$ spectra with $(000) \rightarrow(001)$ and $(001) \rightarrow(002)$ peak spacings of 0.45 and $0.33 \mathrm{eV}$ for $\mathrm{H}_{3} \mathrm{O}^{-}$and 0.34 and $0.29 \mathrm{eV}$ for $\mathrm{D}_{3} \mathrm{O}^{-}$, in excellent agreement with experiment. The peak spacings are somewhat different than those in the $2 \mathrm{D}$ spectra

FIG. 5. Four degree of freedom photodetachment intensities from the $\nu=0$ state of the anion for (a) $\mathrm{H}_{3} \mathrm{O}^{-}$and (b) $\mathrm{D}_{3} \mathrm{O}^{-}$. The anion equilibrium bending angles are $\gamma_{1}^{0}=175.9^{\circ}$ and $\gamma_{2}^{0}=104.8^{\circ}$. The solid line is the total intensity, the dashed line is the product-selected intensity, and the dotdashed line is the reactant-selected intensity.

and the 4D spectra in Fig. 5 indicating that they are sensitive to the constraint to two degrees of freedom and the choice of anion equilibrium angles. The bending progression and broadened peaks seen in the spectra of Fig. 5 have disappeared but there is some asymmetry in the (001) peak in Fig. 6(a) which may be attributed to $\mathrm{OH}$ bending excitation. Asymmetry is not easily discerned in the other peaks and there is not the same degree of asymmetry as observed in the experiment. In these spectra the relative intensity of the (001) peak to the (000) peak is significantly reduced from the 2D spectra and the 4D spectra of Fig. 5. A small amount of reactants is formed in the $\mathrm{H}_{3} \mathrm{O}^{-}$case while no reactants are seen for $\mathrm{D}_{3} \mathrm{O}^{-}$. Again a dip at the energy of $\mathrm{H}_{2}(v=1)$ is observed.

The anion equilibrium angles were chosen to give greater Franck-Condon overlap with the neutral equilibrium geometries. The resulting decrease in bending excitation of 

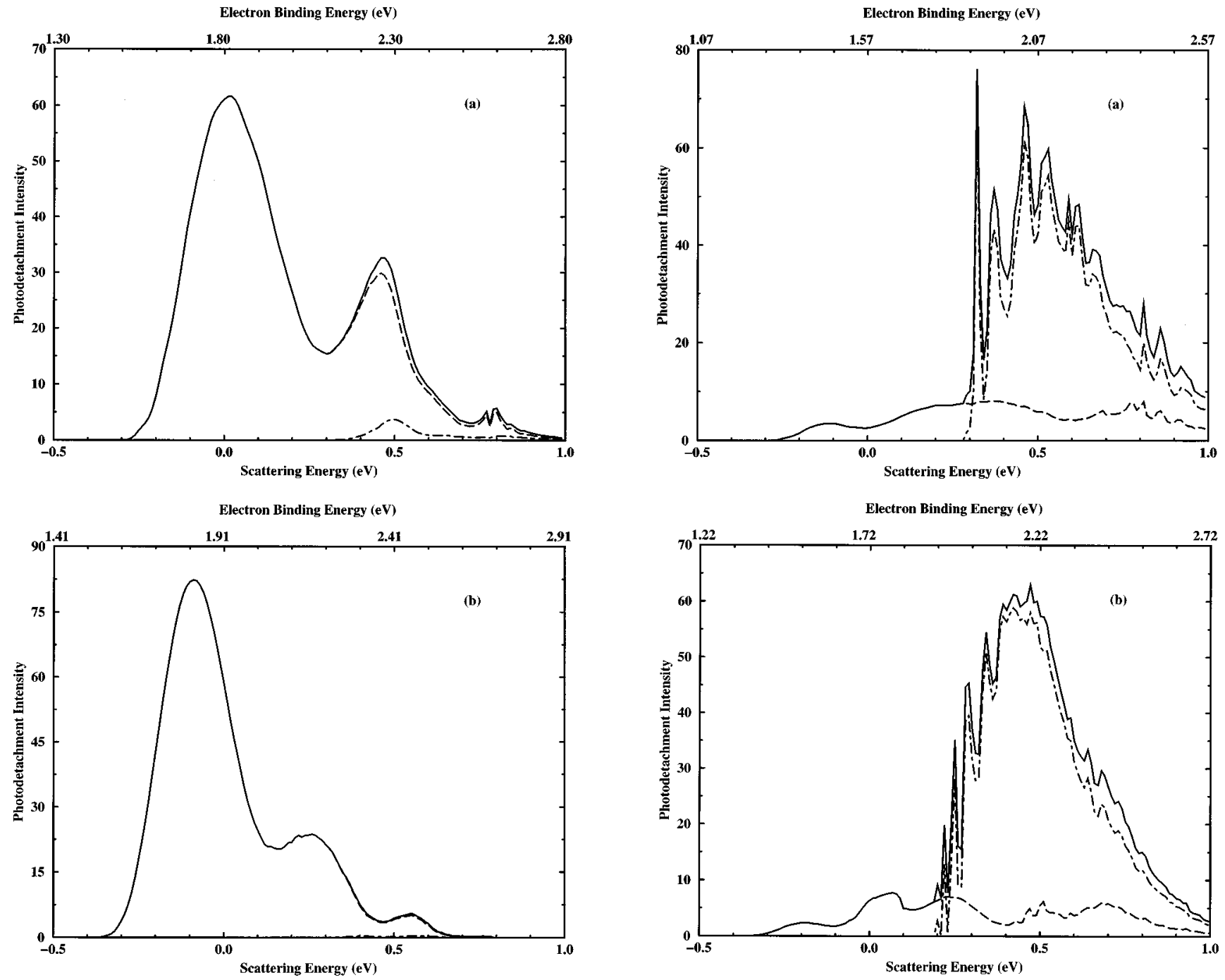

FIG. 6. Same as Fig. 5 except the anion equilibrium bending angles are $\gamma_{1}^{0}=169^{\circ}$ and $\gamma_{2}^{0}=122^{\circ}$.

the neutral gives better agreement with the observed experimental spectra. This indicates that these angles may be more similar in the neutral and anion than is given by the current potential energy surfaces. Comparison of Figs. 5 and 6 indicates that the relative intensities of the peaks are also affected by a change in the equilibrium angles. If the equilibrium angles for the anion are not near those for the neutral, then the region of Franck-Condon overlap sits higher up on the repulsive wall of the neutral potential where the excited vibrational states have greater amplitude.

The spectral features appear at lower electron binding energies than in the 2D calculations. The (000) peak occurs at 1.80 and $1.82 \mathrm{eV} e B E$ for $\mathrm{H}_{3} \mathrm{O}^{-}$and $\mathrm{D}_{3} \mathrm{O}^{-}$, respectively. This is 0.27 and $0.29 \mathrm{eV}$ higher than seen in the experiment. (We should note that this effect is seen in Fig. 5(a) as well with the first peak at $1.83 \mathrm{eV} e B E$.) A difference between the neutral and anion zero point energies in the bending degrees of freedom leads to these lower electron binding energies than seen in the $2 \mathrm{D}$ case.

Figure 7 shows the $\nu=2$ spectrum for (a) $\mathrm{H}_{3} \mathrm{O}^{-}$and (b)
$\mathrm{D}_{3} \mathrm{O}^{-}$with the anion equilibrium angles $\gamma_{1}^{0}=175.9^{\circ}$ and $\gamma_{2}^{0}=104.8^{\circ}$. The same basic structure is seen as in Fig. 4: a narrow peak followed by a very broad feature. There are however, large differences between these spectra and the $2 \mathrm{D}$ results. The narrow peak is much less dominant and in the deuterated spectrum is not identifiable as a separate feature. The broad peak has a bending progression superimposed on it. As in the 2D case, the majority of the intensity leads to the formation of reactants. This anion state has the majority of its Franck-Condon overlap with the $\mathrm{OH}+\mathrm{H}_{2}$ side of the neutral surface leading to these reactant-dominated features. There is still some Franck-Condon overlap on the product side of the neutral surface which is seen as a small background in the spectrum. We note that the broad feature is centered at an electron binding energy of about $2.09 \mathrm{eV}$ for $\mathrm{H}_{3} \mathrm{O}^{-}$and $2.17 \mathrm{eV}$ for $\mathrm{D}_{3} \mathrm{O}^{-}$. These values are 0.04 and 0.12 $\mathrm{eV}$ higher than observed in the experiment.

As discussed by deBeer et al. ${ }^{15}$ the differences in electron binding energies may in part be attributed to the difference between the calculated value of the dissociation energy 
of $\mathrm{H}^{-} \cdots \mathrm{H}_{2} \mathrm{O}$ to give $\mathrm{H}_{2} \mathrm{O}+\mathrm{H}^{-}(0.79 \mathrm{eV})$ and the experimentally measured value $(0.62 \pm 0.04 \mathrm{eV})$. This would put the (000) peak in the $\nu=0$ spectra as $0.10 \mathrm{eV}$ higher than observed in the experiment. The broad peak in the $\nu=2 \mathrm{spec}-$ trum would be $0.13 \mathrm{eV}$ lower than is seen in the experiment. [We note that the calculated spectra have larger peak widths than those in the experiment. Thus the electron binding energies of the onsets of the spectra are closer to the experiment than the peak positions.] deBeer et al. have suggested that the barrier is too "late" in the WDSE surface, and should be moved earlier into the $\mathrm{OH}+\mathrm{H}_{2}$ valley. Our results are consistent with this conclusion. Moving the barrier later would reduce the zero point energy of the $\mathrm{H}_{2} \mathrm{O} \cdots \mathrm{H}$ neutral thus shifting the peak positions to lower energy in the $\nu=0$ spectra. It might also increase the zero point energy of the $\mathrm{HO} \cdots \mathrm{H}_{2}$ neutral thus shifting the broad peak in the $\nu=2$ spectrum to higher energies.

\section{CONCLUDING REMARKS}

We have presented the results of two and (planar) four degree of freedom calculations of the photodetachment spectra of $\mathrm{H}_{3} \mathrm{O}^{-}$and $\mathrm{D}_{3} \mathrm{O}^{-}$. The spectra were computed using a DVR-ABC Green's function which enabled the total and arrangement-selected intensities to be obtained simultaneously. In addition a multiply-shifted quasi-minimal residual (MSQMR) method was used to obtain the Green's function for many energies at once, producing a significant savings in computational time.

The $\mathrm{H}_{3} \mathrm{O}^{-}$anion has two local minima, one of the form $\mathrm{H}_{2} \mathrm{O} \cdots \mathrm{H}^{-}$(the global minimum) which lies under the product side of the neutral surface, and the other of the form $\mathrm{HO}^{-} \cdots \mathrm{H}_{2}$ lying under the reactant side of the neutral surface. As a result the photodetachment spectra are highly sensitive to the initial vibrational state of $\mathrm{H}_{3} \mathrm{O}^{-}$; the $\nu=0$ state is localized in the $\mathrm{H}_{2} \mathrm{O} \cdots \mathrm{H}^{-}$well and results primarily in the formation of products while the $\nu=2$ state is delocalized over both wells and results primarily in the formation of reactants.

We have performed the two degree of freedom calculations to illustrate the basic nature of the spectra and to demonstrate agreement with 2D calculations by de Beer et al. ${ }^{15}$ Our 2D spectra are in good agreement with those of de Beer et al. despite the use of somewhat different coordinates. The four degree of freedom calculations represent our best prediction of the experimental spectra. That is, if the potential energy surfaces we used were the "true" ones, we would expect the 4D spectra to be in excellent agreement with the experiment. Since the spectra are not in excellent agreement, we can analyze the differences to obtain information about the "true" potential energy surfaces.

The $\nu=0$ spectrum consists of three peaks which are assigned to the local mode $\mathrm{OH}$ stretch in the neutral $\mathrm{H}_{2} \mathrm{O}$ of the products. ${ }^{15}$ Our calculated four degree of freedom spectra have this same basic structure. However, in the case where the anion equilibrium bending angles for $\mathrm{OH}$ and $\mathrm{H}_{2}$ are given the values obtained from $a b$ initio results by de Beer et al. the spectrum shows significant bending excitation of the neutral. In addition the relative intensity of the (001) peak to the (000) peak is nearly one. Changing the angles to that of the transition state geometry of the neutral eliminates most of the bending excitation and lowers the intensity of the (001) peak relative to the (000) peak. Since neither the neutral nor anion potential are accurately known, it is not possible to state whether it is the anion or the neutral bending potential which is in error. The conclusion that can be drawn is that the angles are more similar in the two potentials than given by the current surfaces.

The $\nu=2$ spectrum is dominated by a broad peak leading to the formation of reactants. The large narrow peak which was observed in the (2D) calculations, is much less dominant in the (4D) spectra. The calculated intensities are in good agreement with the experimental spectra assigned to the $\mathrm{HO}^{-} \cdots \mathrm{H}_{2}$ anion minimum. The theoretical spectrum does have a small background leading to products due to the delocalized nature of the $\nu=2$ eigenstate across both anion minima.

Finally, the theoretical calculations give the electron binding energy of the $\nu=0$ spectra as too high relative to the experimental results. While for the $\nu=2$ spectra, the electron binding energy is too low compared to experiment. This disagreement between the theoretical and experimental results is consistent with the suggestion by de Beer et al. ${ }^{15}$ that the barrier on the neutral WDSE potential energy surface is too "late" and should be moved earlier into the $\mathrm{OH}+\mathrm{H}_{2}$ valley.

Note added in proof. Since the submission of this paper we have become aware of new $a b$ initio calculations on the $\mathrm{H}_{3} \mathrm{O}^{-}$potential. Results at the $\operatorname{CCSD}(\mathrm{T})$ aug-cc-pVTZ level $^{29}$ give equilibrium geometries in good agreement with those of de Beer et al. ${ }^{15}$ This indicates that it is the equilibrium angles in the neutral potential (particularly the $\mathrm{H}-\mathrm{O}-\mathrm{H}$ angle) that are in error.

\section{ACKNOWLEDGMENTS}

We wish to thank Professor Daniel M. Neumark and Dr. Esther de Beer for many useful discussions and for providing the anion potential energy surface. This work was supported by the Director, Office of Energy Research, Office of Basic Energy Sciences, Chemical Sciences Division of the U.S. Department of Energy under Contract No. DE-AC0376SF00098. W.H.T. acknowledges support from the National Science Foundation. H.O.K. acknowledges a grant from the Swedish Fulbright Commission and support from NSF grant CHE 94-22559.

${ }^{1}$ D. M. Neumark, Acc. Chem. Res. 26, 33 (1993); R. B. Metz, S. E. Bradforth, and D. M. Neumark, Adv. Chem. Phys. 81, 1 (1992).

${ }^{2}$ See G. C. Schatz, J. Phys. Chem. 94, 6157 (1990), and references therein. ${ }^{3}$ See, D. E. Manolopoulos, K. Stark, H.-J. Werner, D. W. Arnold, S. E. Bradforth, and D. M. Neumark, Science 262, 1852 (1993), and references therein.

${ }^{4}$ D. C. Clary, J. Chem. Phys. 95, 7298 (1991).

${ }^{5}$ D. C. Clary, J. Chem. Phys. 96, 3656 (1992); Chem. Phys. Lett. 192, 34 (1992).

${ }^{6}$ G. Nyman and D. C. Clary, J. Chem. Phys. 99, 7774 (1993)

${ }^{7}$ G. Nyman and D. C. Clary, J. Chem. Phys. 101, 5756 (1994).

${ }^{8}$ J. Echave and D. C. Clary, J. Chem. Phys. 100, 402 (1994).

${ }^{9}$ J. M. Bowman and D. Wang, J. Chem. Phys. 96, 7852 (1992); D. Wang 
and J. M. Bowman, ibid 96, 8906 (1992); 98, 6235 (1992).

${ }^{10}$ H. Szichman, I. Last, A. Baram, and M. Baer, J. Phys. Chem. 97, 6436 (1993); H. Szichman, I. Last, and M. Baer, ibid 98, 828 (1994).

${ }^{11}$ D. Zhang and J. Z. H. Zhang, J. Chem. Phys. 100, 2697 (1994).

${ }^{12}$ D. Zhang and J. Z. H. Zhang, J. Chem. Phys. 101, 1146 (1994).

${ }^{13}$ D. Neuhauser, J. Chem. Phys. 100, 9272 (1994).

${ }^{14}$ U. Manthe, T. Seideman, and W. H. Miller, J. Chem. Phys. 99, 10078 (1994); 101, 4759 (1994).

${ }^{15}$ E. de Beer, E. H. Kim, D. M. Neumark, R. F. Gunion, and W. C. Lineberger, J. Phys. Chem. 99, 13627 (1995).

${ }^{16}$ G. C. Schatz and H. Elgersma, Chem. Phys. Lett. 73, 21 (1980).

${ }^{17}$ S. S. Xantheas and T. H. Dunning, Jr., J. Chin. Chem. Soc. 42, 241 (1995).

${ }^{18}$ D. O. Harris, G. G. Engerholm, and W. D. Gwinn, J. Chem. Phys. 43, 1515 (1965).

${ }^{19}$ A. S. Dickinson and P. R. Certain, J. Chem. Phys. 49, 4209 (1963).
${ }^{20}$ J. V. Lill, G. A. Parker, and J. C. Light, Chem. Phys. Lett. 89, 483 (1982); J. C. Light, I. P. Hamilton, and J. V. Lill, J. Chem. Phys. 82, 1400 (1985); Z. Bačić and J. C. Light, ibid 85, 4594 (1986); R. M. Whitnell and J. C. Light, ibid 89, 3674 (1988); S. E. Choi and J. C. Light, ibid 92, 2129 (1990).

${ }^{21}$ W. H. Thompson and W. H. Miller, J. Chem. Phys. 101, 8620 (1994), and references therein.

${ }^{22}$ S. P. Walch and T. H. Dunning, Jr., J. Chem. Phys. 72, 1303 (1980).

${ }^{23}$ D. T. Colbert and W. H. Miller, J. Chem. Phys. 96, 1982 (1992).

${ }^{24}$ Y. Saad and M. H. Schultz, SIAM J. Sci. Stat. Comput. 7, 856 (1986).

${ }^{25}$ R. W. Freund and N. M. Nachtigal, Numer. Math. 60, 315 (1991).

${ }^{26}$ H. O. Karlsson, J. Chem. Phys. 103, 4914 (1995).

${ }^{27}$ R. W. Freund, in Numerical Linear Algebra, edited by L. Reichel, A. Ruttan and R. Varga (de Gruyter, Berlin, 1993).

${ }^{28}$ B. N. Datta and Y. Saad, Linear Algebra Appl. 154-156, 225 (1991).

${ }^{29} \mathrm{~S}$. L. Mielke (private communication). 